\title{
Computer-assisted method for compiling a blood group register
}

\author{
L. A. BEllchambers, M. G. RinSler, AND A. G. SIGNY \\ From the Group Laboratory, St Stephen's Hospital, London
}

SYNOPSIS The compilation of the records of a serology department using a computer to produce alphabetically ordered files is described. This has proved to be an efficient and economical technique which may well be applied to other similar problems in a large clinical laboratory.

As more technical procedures tend to be automated and the flow of data increases, the problems of consolidating and extracting information in clinical laboratories are becoming more acute. Although considerable thought has been given to cumulative individual records (Whitby and Owen, 1965; Flynn and Vernon, 1965), little attention has been given to the working files of data within the laboratory.

The present filing system in this laboratory involves sorting by hand the NCR copies of reports in alphabetical order of patients' names, so leading to large, unwieldy files. The files require considerable clerical time to keep in order as cards are frequently lost or misfiled. Withdrawal and re-entry of records is time consuming. Because of these difficulties we have undertaken an experiment in the application of modern techniques in automated data handling to the problems in the clinical laboratory. We chose the records of the serological division of the haematology department as the most suitable for investigation. This file, which is separated from the main haematology file, contains the records of the transfusion work of the laboratory, antenatal serology, and general serology in relation to other haematological problems. Each year this file grows by 10,000 records. The type of test performed is limited in number and fairly uniformly applied to each specimen and includes $\mathrm{ABO}$ grouping, $\mathrm{Rh}$ grouping, and the detection of antibodies. This file is frequently referred to for checking current against previous data and accurate up-to-date information is essential. Our aim, therefore, has been to produce an alphabetically listed file which could be sorted and printed by computer, was convenient to handle, and occupied minimal space. Furthermore, since the cost of producing the file should be within reasonable Received for publication 20 March 1968. financial limits, we proposed to spend not more than the salary of a half-time clerical officer per annum on the system.

Our first problem was to decide what the information to be stored and printed should be, within the limits of the electric page printer. This information was divided into patient and sample identification data, clinical data, and laboratory data. The layout we adopted is shown in Table I. In all there were 114 characters, excluding spaces and tabulation, of which 49 were identification characters including name, hospital number, sample number, date, and location of the patient. The program for the production of the register was based on the flow chart presented in Fig. 1, and written by members of Elliott Medical Automation Ltd for the operation of the Elliott 4100 computer at the Computer Centre, Royal Postgraduate Medical School, Hammersmith.

Special laboratory worksheets were printed on NCR paper so that the identification data, working data, and results could be recorded on one line. After the entry of the working data and results the upper sheet was preserved in the laboratory for immediate reference. The copy of the work sheet is given to the typist whose task is to produce an accurate perforated paper tape in a format that the computer can use. This work is carried out on a page printer with a perforated paper punch. The printed page is checked visually for typographical errors and the paper tapes are ready to be fed into the computer. The unsorted file is read from the paper tape into the computer store and from there is written on to a magnetic tape. This unsorted file of about two weeks' work is then sorted alphabetically by program and added to the sorted file on magnetic tape. A perforated paper tape corresponding to two weeks' sorted file is produced by the computer and 
TABLE I

REPRODUCTION OF THE PRINT-OUT OF THE HAEMATOLOGY REGISTER

\begin{tabular}{|c|c|c|c|c|c|c|c|}
\hline \multirow[b]{2}{*}{ Date } & \multicolumn{6}{|c|}{ REPRODUCTION OF THE PRINT-OUT OF THE HAEMATOLOGY REGISTER } & \multirow{2}{*}{ Hospit } \\
\hline & $\begin{array}{l}\text { Laboratory } \\
\text { Number }\end{array}$ & Name & $\begin{array}{l}\text { Hospital } \\
\text { Number }\end{array}$ & Age & $\operatorname{Sex}$ & Ward & \\
\hline $18 / 08 / 67$ & 07081 & Smart, Judith & & 020 & $\mathbf{F}$ & $\mathbf{M T}$ & SMA \\
\hline $19 / 05 / 67$ & 04433 & Smart, June & & 020 & $\mathbf{F}$ & $\mathbf{M T}$ & SMA \\
\hline $06 / 04 / 67$ & 03029 & Smart, Patricia & 139529 & 026 & $\mathbf{F}$ & $\mathbf{R H}$ & SMA \\
\hline $01 / 04 / 67$ & 02900 & Smart, Rosemary & & 020 & $\mathbf{F}$ & MT & SMA \\
\hline $14 / 09 / 67$ & 07906 & Smart, Rosemary & & 020 & $\mathbf{F}$ & MT & SMA \\
\hline $24 / 10 / 67$ & 09263 & Smart, Rosemary & & C & & MT & SMA \\
\hline $03 / 05 / 67$ & 03916 & Smart, Vickie & A039710 & 020 & $\mathbf{F}$ & MT & SSH \\
\hline $26 / 07 / 67$ & 06442 & Smith, Barbara & A023005 & 027 & $\mathbf{F}$ & MT & SSH \\
\hline $23 / 08 / 67$ & 07216 & Smith, Christina & 161630 & & $\mathbf{F}$ & $\mathbf{R H}$ & SMA \\
\hline $23 / 06 / 67$ & 05471 & Smith, Christine & & 026 & $\mathbf{F}$ & MT & SMA \\
\hline $22 / 06 / 67$ & 05451 & Smith, Christophe & & 016 & $\mathbf{M}$ & & PUT \\
\hline $09 / 10 / 67$ & 08733 & Smith, Hilda & 037619 & 068 & $\mathbf{F}$ & $5 B$ & SSH \\
\hline $18 / 10 / 67$ & 09009 & Smith, Patricia & A043430 & 026 & $\mathbf{F}$ & MT & SSH \\
\hline $29 / 05 / 67$ & 04726 & Smith, Phyllis & 026232 & 064 & $\mathbf{F}$ & 6B & SSH \\
\hline $19 / 09 / 67$ & 08045 & Smith, Vera & A004041 & 029 & $\mathbf{F}$ & $5 C$ & SSH \\
\hline $15 / 05 / 67$ & 04284 & Smith, Violet & & & $\mathbf{F}$ & $5 C$ & SSH \\
\hline $19 / 09 / 67$ & 08047 & Smythe, Alice & & 039 & $\mathbf{F}$ & $\mathbf{R H}$ & SMA \\
\hline $23 / 06 / 67$ & 05464 & Smythe, Baby & & C & & MT & SMA \\
\hline $03 / 06 / 67$ & 04870 & Smythe, Gladys & 005007 & 080 & $\mathbf{F}$ & $\mathbf{H N}$ & PBH \\
\hline $12 / 10 / 67$ & 08832 & Snag, Florence & A042095 & 075 & $\mathbf{F}$ & $3 \mathrm{C}$ & SSH \\
\hline $15 / 07 / 67$ & 06141 & Snag, Nicola & & 023 & $\mathbf{F}$ & & PUT \\
\hline $04 / 08 / 67$ & 06691 & Sneath, Rita & 132159 & 029 & $\mathbf{F}$ & $\mathbf{M T}$ & SMA \\
\hline $13 / 09 / 67$ & 07837 & Sneath, Susan & A042612 & 018 & $\mathbf{F}$ & MT & SSH \\
\hline $22 / 05 / 67$ & 04509 & Snell, David & 005305 & 024 & $\mathbf{F}$ & MT & PBH \\
\hline $12 / 05 / 67$ & 04198 & Snell, Peter & & 031 & $\mathbf{F}$ & MT & SMA \\
\hline $27 / 07 / 67$ & 06474 & Snook, Edward & & 068 & $\mathbf{M}$ & $\mathbf{A C}$ & SSH \\
\hline $15 / 08 / 67$ & 06968 & Sober, Joan & A003692 & 026 & $\mathbf{F}$ & $5 C$ & SSH \\
\hline $31 / 07 / 67$ & 06592 & Sofer, Amanda & & $\mathbf{N}$ & $\mathbf{F}$ & VT & SMA \\
\hline $28 / 07 / 67$ & 06502 & Soft, Patrick & & 026 & $\mathbf{F}$ & MT & SMA \\
\hline $19 / 05 / 67$ & 04432 & Sole, Mary & & 034 & $\mathbf{F}$ & MT & SMA \\
\hline $23 / 10 / 67$ & 09125 & Solid, Baby & & $\mathbf{C}$ & $\mathbf{F}$ & MT & SSH \\
\hline $31 / 08 / 67$ & 07425 & Solid, Sheila & A026885 & 033 & $\mathbf{F}$ & MT & SSH \\
\hline $14 / 09 / 67$ & 07897 & Solid, Sheila & A026885 & 033 & $\mathbf{F}$ & MT & SSH \\
\hline $12 / 10 / 67$ & 08808 & Solid, Sheila & A026885 & 033 & $\mathbf{F}$ & MT & SSH \\
\hline $30 / 09 / 67$ & 08317 & Solid, Sheila & A026885 & 033 & $\mathbf{F}$ & MT & SSH \\
\hline $17 / 05 / 67$ & 04333 & Solid, Sheila & A026885 & 033 & $\mathbf{F}$ & MT & SSH \\
\hline $26 / 06 / 67$ & 05538 & Somers, Olive & 004994 & 023 & $\mathbf{F}$ & MT & PBH \\
\hline $27 / 07 / 67$ & 06477 & Soper, Marion & & 024 & $\mathbf{F}$ & TE & SMA \\
\hline
\end{tabular}

this is printed off-line with a page printer. The sorted records on the magnetic tape file are now merged with the previously sorted records. At intervals of six months the entire file is printed either on a page printer from a perforated paper tape or directly by a line printer. Since each record occupies one line compared with a whole card there is a considerable saving of space. The records of each patient are accumulated on successive lines which facilitates reading and comparisons and there is no need to draw and replace filing cards.

CODING OF DIAGNOSTIC AND TRANSFUSION DATA

Must be one of the following:

$$
\begin{aligned}
& \mathbf{T}+\text { number } \\
& \mathbf{T}=\text { Transfusion } \\
& \left.\begin{array}{rl}
\mathbf{M}+\text { number }
\end{array}\right\} \text { eg P4, P1/TO, P4/M2/T3 } \quad \begin{array}{l}
\mathbf{P}=\text { Pregnancy } \\
\mathbf{M}=\text { Miscarriage }
\end{array} \\
& \text { Maximum number of characters is } 8 \text {, including the obliques. } \\
& \text { S = surgery GI = gastrointestinal } \\
& \text { HA = haemolytic anaemia } \\
& \text { PA = pernicious anaemia UC = ulcerative colitis } \\
& \text { LEU = leukaemia CDA = coagulation defect } \\
& \text { AP = aplastic anaemia due to drugs } \\
& \text { GY = gynaecology } \\
& \text { TH = thalassaemia } \quad \text { TA } \quad \text { = threatened abortion } \\
& \text { CD = coagulation defect } \quad \text { OB }=\text { miscellaneous }
\end{aligned}
$$


TABLE I-continued

REPRODUCTION OF THE PRINT-OUT OF THE HAEMATOLOGY REGISTER

\begin{tabular}{|c|c|c|c|c|c|c|c|c|c|c|c|}
\hline History & $A B O$ & $\begin{array}{l}\text { Rh } \\
\text { Factor }\end{array}$ & Genotype & Antibodies & $\begin{array}{l}\text { Direct } \\
\text { Coombs }\end{array}$ & $\begin{array}{l}\text { Antiboc } \\
\text { Saline }\end{array}$ & $\begin{array}{r}\text { titre } \\
\text { Alb }\end{array}$ & Coombs & Ig & $V D R L$ & Bottle Numbers \\
\hline P1 & $\mathbf{A}$ & POS & & NIL & & & & & & NEG & \\
\hline \multirow[t]{2}{*}{ P1 } & $\mathbf{A}$ & POS & & NIL & & & & & & NEG & \\
\hline & $\mathbf{A}$ & POS & & NIL & & & & & & NEG & \\
\hline \multirow[t]{3}{*}{ P1 } & $\mathbf{O}$ & NEG & LRLR & NIL & & & & & & NEG & \\
\hline & $\mathbf{O}$ & NEG & & NIL & & & & & & NEG & \\
\hline & o & POS & & NIL & NEG & & & & & NEG & \\
\hline P1 & $\mathbf{O}$ & NEG & LRLR & NIL & & & & & & NEG & \\
\hline P3 & $\mathbf{A}$ & POS & & NIL & & & & & & NEG & \\
\hline OB & B & NEG & LRLR & D & & NIL & 0008 & 0016 & G & NEG & \\
\hline \multirow[t]{2}{*}{ P2 } & $\mathbf{A}$ & POS & & NIL & & & & & & NEG & \\
\hline & $\mathbf{A}$ & POS & & NIL & & & & & & REP & \\
\hline GY & B & POS & & NIL & & & & & & NEG & \\
\hline P1 & $\mathbf{O}$ & POS & & NIL & & & & & & NEG & \\
\hline \multirow[t]{2}{*}{ P7/M1 } & $\mathbf{A}$ & POS & & NIL & & & & & & NEG & \\
\hline & $\mathbf{A}$ & POS & & NIL & & & & & & NEG & \\
\hline \multirow[t]{2}{*}{$\mathbf{S}$} & $\mathbf{O}$ & POS & & NIL & & & & & & NEG & $040658040682 \mathrm{~N}$ \\
\hline & $\mathbf{A}$ & POS & & NIL & & & & & & REP & MM \\
\hline \multirow[t]{2}{*}{ TA } & $\mathbf{O}$ & POS & & NIL & & & & & & NEG & \\
\hline & $\mathbf{A}$ & POS & & NIL & NEG & & & & & NEG & \\
\hline $\mathbf{S}$ & o & POS & & NIL & & & & & & NEG & 042242043204 \\
\hline $\mathbf{S}$ & O & POS & & NIL & & & & & & NEG & 055201058681 \\
\hline TA & $\mathbf{A}$ & POS & & NIL & & & & & & NEG & 078258078259 \\
\hline P3/M1 & o & POS & & NIL & & & & & & NEG & \\
\hline P1 & $\mathbf{O}$ & NEG & LRLR & NIL & & & & & & NEG & \\
\hline P1 & B & POS & & NIL & & & & & & NEG & \\
\hline P2 & $\mathbf{A B}$ & POS & & NIL & & & & & & NEG & \\
\hline CDA & $\mathbf{o}$ & NEG & LRLR & NIL & & & & & & NEG & \\
\hline \multirow[t]{2}{*}{$\mathbf{A D}$} & $\mathbf{A}$ & POS & & NIL & & & & & & NEG & $005506-$ \\
\hline & $\mathbf{A}$ & NEG & LRLR & NIL & & & & & & NEG & 017862017864 \\
\hline \multirow[t]{2}{*}{$\mathbf{A D}$} & $\mathbf{A}$ & POS & & NIL & & & & & & REP & $\begin{array}{l}008557008620- \\
008566-\end{array}$ \\
\hline & B & POS & & NIL & & & & & & REP & 007114 \\
\hline P1 & B & POS & & NIL & & & & & & NEG & \\
\hline \multirow[t]{6}{*}{ P4 } & $\mathbf{A}$ & POS & & NIL & & & & & & NEG & \\
\hline & o & POS & & NIL & NEG & & & & & NEG & \\
\hline & O & NEG & & NIL & & & & & & NEG & \\
\hline & o & NEG & & NIL & & & & & & NEG & \\
\hline & o & NEG & & NIL & & & & & & NEG & \\
\hline & o & NEG & & NIL & & & & & & NEG & \\
\hline P6 & o & NEG & LRLR & NIL & & & & & & NEG & \\
\hline P1 & $\mathbf{O}$ & POS & & NIL & & & & & & NEG & \\
\hline $\mathbf{S}$ & $\mathbf{A}$ & POS & & NIL & & & & & & REP & $\begin{array}{l}000995001016- \\
077865 \mathrm{~N}\end{array}$ \\
\hline
\end{tabular}

CODING OF ANTIBODY DATA

CODFNG OF ABO GROUPS

Must be one of the following:

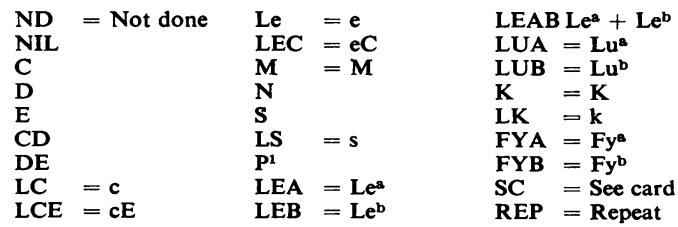

CODING OF RH GROUP FACTORS

Must be POS, NEG, DU or REP

$\mathbf{R E P}=$ Repeat

Must be one of the following:

$$
\begin{aligned}
& \text { A, A1, A2, A3, A4, AM } \\
& \text { AB, A1B, A2B, A3B } \\
& \text { B, O } \\
& \text { RHESUS (G = GENOTYPE) }
\end{aligned}
$$

Must be any pair of the following:

R1, R2, RO, RZ, RC, RE, RY, LR

$\mathbf{R C}=\mathbf{R}^{\mathbf{1}}$

$R E=R^{11}$

$\mathbf{L R}=\mathbf{r}$ 


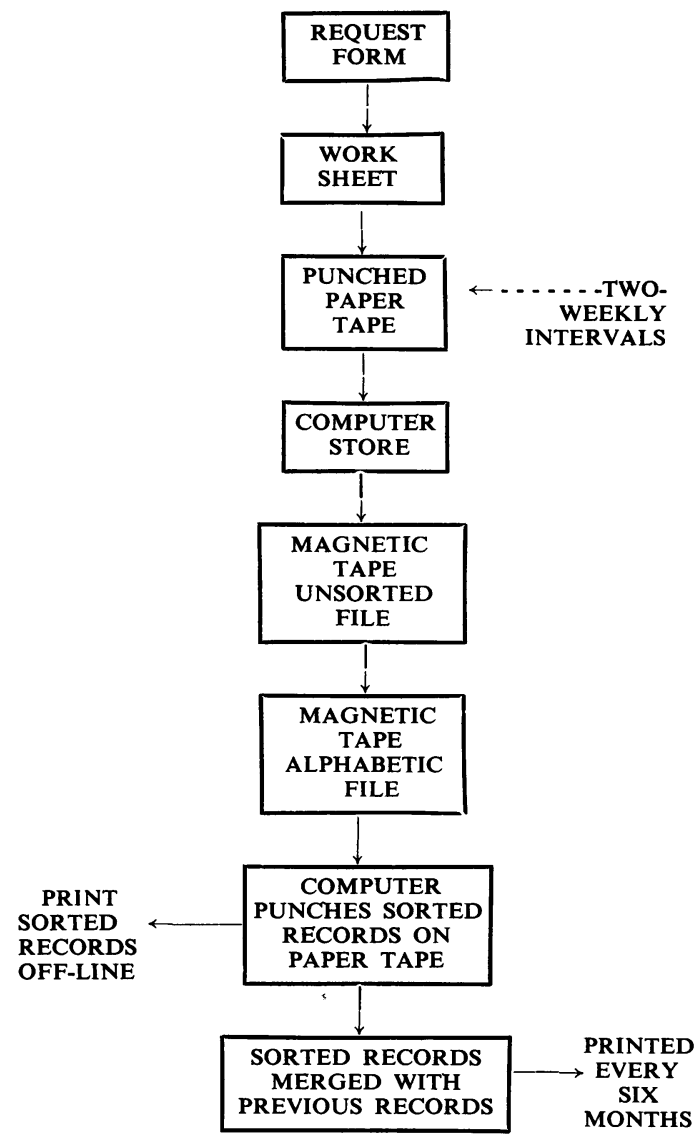

FIG. 1. Reproduction of the flow chart of the haematology register.
Since a full description of the information in eacen column would require very lengthy records, coding of information was performed as far as possible. We have endeavoured to devise a code which resembled conventional abbreviations, either locet or national, to simplify interpretation of these print-out. Examples are shown below.

The main difficulties of the new system have been chiefly in the transfer of information from one document to another. Careful visual checks are applied to the completed worksheet and to the first printing of the unsorted data and all correctiogs entered on the perforated paper tape. After the first run through the computer, records which are nit accepted are corrected and entered again. Typis time is about 50 to 60 records an hour to whisb correction time must be added. To speed the tine of printout, particularly of the six-monthly corgpilation, a line printer has been employed which cuts the printing time to about $5 \%$ of that of a pase printer which types one character at a time.

With greater experience we would expect produce a more compact, efficient program and extend the program so that certain types of da: can be extracted by the computer: for example the list of primiparous patients who are $D$ negatie now being produced manually in order to forecast the immunoglobulin requirements for the patients. Such applications will greatly increase the usefulness of the classified information.

\section{REFERENCES}

Flynn, F. V., and Vernon, J. (1965). J. clin. Path., 18, 678. Whitby, L. G., and Owen, J. A. (1965). Ibid., 18, 668. 